\title{
ZONIFICACIÓN DEL RIESGO POR INUNDACIÓN COMO APORTE A LA PREVENCIÓN, EN EL PUERTO - LAGUNA DE LA COCHA- COLOMBIA
}

\section{Zoning of flood risk as a contribution to prevention, in the port - Laguna de la Cocha- Colombia.}

\author{
Juan Guillermo Delgado Noguera \\ Corporación Autónoma regional de Nariño (Corponariño). \\ Geólogo.jdelgadonoguera@gmail.com
}

\section{Resumen}

En el ordenamiento y planificación del territorio de zonas propensas a inundaciones, la gestión del riesgo debe ser parte integral y la base para la toma de decisiones, que armonicen la prevención y el bienestar en el contexto de un desarrollo sostenible. El presente estudio permitió determinar la zonificación del riesgo de desastre por inundaciones en el caserío del Puerto - Laguna de La Cocha, Colombia, con el objeto de generar pautas para el ordenamiento territorial. Para ello se llevó a cabo un análisis del comportamiento histórico de la variable hidrológica, se ajustó una distribución estadística y estimaron niveles para diferentes periodos de retorno adoptados. Estos se convierten en cotas para llevarlos a planos topográficos, y así con base en criterios hidrológicos y geomorfológicos, definir distintas zonas bajo amenaza. Con base en la identificación de infraestructura e instalaciones críticas presentes en el área, se evaluó la vulnerabilidad, y a partir de la combinación de zonas de amenaza y vulnerabilidad se establecieron tres zonas del riesgo por inundación: alto, medio y bajo. Determinadas las categorías de riesgo, se generaron medidas de prevención y ordenamiento territorial, para zonas de alto y mediano riesgo con probabilidad alta de ocurrencia de inundaciones, se aconseja prohibir los asentamientos de viviendas, en estos sectores, pues

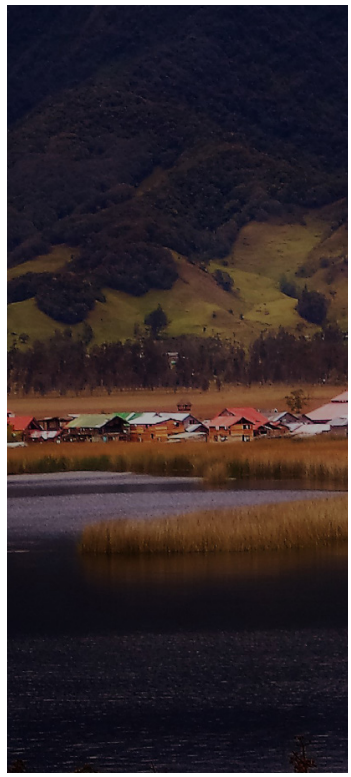

Historia del artículo

Fecha de recepción : $02 / 09 / 2019$ Fecha de aceptación: $15 / 11 / 2019$ 
pueden ocurrir efectos potencialmente dañinos, dada la exposición de personas, infraestructura e instalaciones críticas. Para la zona de bajo riesgo, apoyados en el principio de prevención y sostenibilidad, se sugiere evitar que sea poblado, y así impedir aumentar el grado de riesgo.

Palabras clave: Amenaza, Criterio hidrológico, Guamués, Niveles, Riesgo, Vulnerabilidad.

\section{Abstract}

In the planning and planning of the territory of flood-prone areas, risk management must be an integral part and the basis for decision-making, which harmonize prevention and well-being in the context of sustainable development. The present study allowed determining the zoning of the risk of flood disaster in the hamlet of the Port Laguna de La Cocha, Colombia, in order to generate guidelines for land use planning. For this, an analysis of the historical behavior of the hydrological variable was carried out, a statistical distribution was adjusted and levels were estimated for different return periods adopted. These become levels to take them to topographic planes, and thus based on hydrological and geomorphological criteria, define different areas under threat. Based on the identification of infrastructure and critical facilities present in the area, vulnerability was assessed, and from the combination of threat and vulnerability zones, three flood risk zones were established: high, medium and low. Determined risk categories were generated measures of prevention and territorial planning, for high and medium risk areas with high probability of occurrence of floods, it is advisable to prohibit housing settlements, in these sectors potentially harmful effects may occur, given the exposure of people, infrastructure and critical facilities. For the low risk area, supported by the principle of prevention and sustainability, it is suggested to avoid being populated, and thus prevent increasing the degree of risk.

Keywords: Threat, Hydrological criteria, Guamués, Levels, Risk, Vulnerability.

\section{Introducción}

Dada la gran importancia que tiene la evaluación de eventos extremos de crecidas de distintos cuerpos de agua para la gestión del riesgo de desastres (Ley 1523, 2012) y para un adecuado ordenamiento del territorio (Ley 388, 1997), y teniendo en cuenta las experiencias vividas en las diferentes emergencias en época de lluvias intensas en el caserío El Puerto, del corregimiento El Encano, ubicado en la margen Nor-Oriental de la laguna de La Cocha, municipio de Pasto, departamento de Nariño, Colombia, se hace necesaria la evaluación de dichos eventos y la delimitación de aquellas zonas que son propensas a inundaciones, con el fin de darles un uso adecuado que permita tomar las medidas preventivas necesarias antes del desencadenamiento de dichos eventos.

Es necesario tener en cuenta que El lago Guamués o laguna de La Cocha es un gran embalse natural, segundo en relevancia en Colombia después del lago de Tota 
(Departamento de Boyacá). La Cocha es de gran significancia a nivel nacional y regional, puesto que el país lo inscribió como humedal de jerarquía internacional dentro del convenio RAMSAR en el año 200o, mediante el Decreto 698 del 18 de abril (Ministerio del Ambiente y Desarrollo Sostenible - Colombia).

La precipitación es un evento del clima que condiciona las inundaciones, la naturaleza de los eventos meteorológicos perjudiciales para el área de estudio no son de ocurrencia única, por el contrario, se repiten periódicamente con intensidad variable. En el sector del Puerto de El Encano, los anegamientos se presentan en los meses de julio y agosto. En el sector del Puerto, no existe una delimitación clara a una escala adecuada de diferentes franjas de anegamiento, y en la actualidad se desarrolla una colonización espontánea sin control, que invade áreas de conservación en detrimento del medio ambiente y que coloca en riesgo a la población que se asienta en zonas propensas a inundación.

En cuanto al estudio efectuado, se realizó un análisis multitemporal de la información básica hidrológica (niveles de la laguna) conducente a determinar diferentes zonas de anegamiento en el sector El Puerto - La Cocha, donde los niveles del lago cambian de acuerdo con la alternancia de los períodos de lluvias y períodos secos. Se utilizó las series de datos meteorológicos y niveles diarios del lago en el período 1993-2015 del Instituto de hidrología, meteorología y estudios ambientales de Colombia - IDEAM.
Para el análisis de la variable hidrológica se ajustó una distribución a los datos de niveles máximos anuales, que permitió asociar recurrencias a los eventos extremos observados, caracterizar su distribución temporal y de forma subsiguiente determinar y mapear zonas de inundación. Esta información, sirvió para generar medidas para la prevención y ordenamiento del territorio y se constituye en el objetivo general del presente estudio, de igual manera; esta investigación podrá ser utilizada por el municipio de Pasto como insumo en un proceso de ordenamiento y gestión del riesgo de inundación.

\section{Materiales y Método}

\section{Área de estudio}

La laguna de La Cocha está localizada en la zona andina al Sur Occidente del territorio Colombiano, a una altura aproximada de $2.782 \mathrm{msnm}$, entre las coordenadas $1^{\circ}$ o $1^{\prime} \mathrm{o}$ "

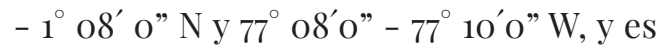
parte del Corredor Andino Amazónico Norte, Ecorregión Bordoncillo Patascoy. La Cocha está ubicada aproximadamente a 22 km de la zona urbana del municipio de Pasto. Tiene un área aproximada de $45 \mathrm{~km}^{2}$, una profundidad máxima de $70 \mathrm{~m}$, el régimen de lluvias es unimodal con una temporada de lluvias intensas de abril a agosto y de pocas lluvias de septiembre a marzo. En la Figura 1, se observa la ubicación del área de estudio (Corponariño, 2011).

Esta área geográfica experimenta cada año, desde diciembre hasta marzo, vientos 

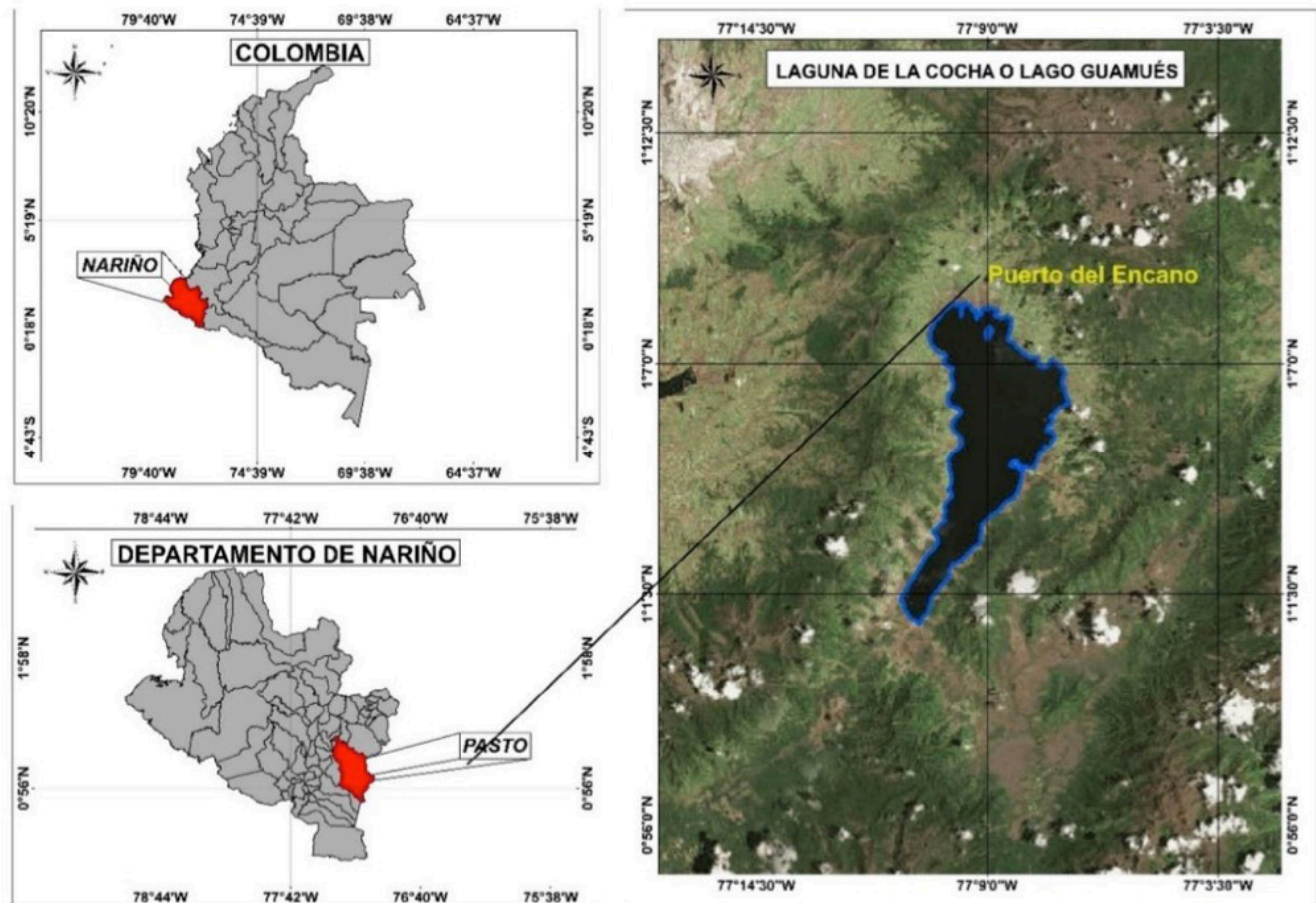

Figura 1. Localización del área de estudio. Fuente: Corponariño

alisios fuertes provenientes del Suroeste (SW), debido a que la Zona de Confluencia Intertropical (ZCIT) se encuentra en su posición más meridional, lo cual ocasiona escasas lluvias dado los constantes desplazamientos nubosos hacia el hemisferio Norte. Por otra parte, durante abril - agosto circula entre las altas montañas cercanas al lago Guamués, una corriente atmosférica muy húmeda llamada masa continental o amazónica; dicho fenómeno físico ocasiona importantes cambios climáticos porque aumenta la precipitación y disminuye el brillo solar, provocando bajas temperaturas para ese período (Instituto Alexander Von Humboldt et al., 2004).
La cuenca alta del río Guamués presenta lluvias alrededor de los $1.300 \mathrm{~mm}$ anuales en el sector de El Encano (Corponariño, 2011). El promedio histórico de precipitación mensual para el período considerado entre 1993 y 2015 es de $115,5 \mathrm{~mm}$; los promedios mensuales y la media anual obtenidos de la serie diaria de niveles del lago para este periodo de los registros del IDEAM en la estación limnimétrica Sindamanoy, alcanzan un nivel promedio histórico de $267 \mathrm{~cm}$ y los máximos niveles se presentan en julio y agosto.

La morfogénesis del Humedal Lacustre lago Guamués (área donde se encuentra el caserío El Puerto de El Encano), es tectónica del 
Terciario y glaciar del cuaternario (Ghul, 1976). Según sus características fisiogeográficas se identifican dos unidades paisajísticas en el área de influencia: Montaña y Altiplano. El paisaje de montaña corresponde a las laderas y vertientes del área de abastecimiento que rodea el humedal lacustre, con formas de modelado glaciar y pendientes suaves hacia la margen oriental y de formas más abruptas y altas pendientes hacia la occidental. Este paisaje influye en las inclinaciones de las cuencas que conforman el humedal; la pendiente de la cuenca determina la velocidad con la que se desarrolla la escorrentía superficial, y el tiempo que lleva el agua de lluvia para concentrarse en los lechos fluviales que constituyen la red de drenaje (Corponariño, 2011).

\section{Determinación de la relación Amenaza -} Vulnerabilidad y riesgo

Existen diferentes metodologías para la elaboración de mapas de riesgo por inundaciones, las técnicas o procesos para la realización de estos mapas se analizan a partir de la combinación de los resultados de las variables de amenaza y vulnerabilidad; de acuerdo a estos criterios y a la disponibilidad de información se desarrollan distintos métodos.

Según Lluís Ribera Masgrau (2004), el riesgo se puede formular como el producto del área afectada, la peligrosidad del suceso, el número de elementos que están en juego (exposición) y su vulnerabilidad.

El riesgo, se define como la combinación de una probabilidad de presentación de un determinado evento, llamado peligro, y las potenciales consecuencias adversas que tendría este evento para la salud humana, el medio ambiente, el patrimonio cultural o las actividades económicas. Normalmente, el riesgo se expresa como Riesgo = Amenaza $\mathbf{x}$ Vulnerabilidad. En general, las unidades en que se estima el riesgo están vinculadas a las unidades de las consecuencias estimadas, divididas por una unidad de tiempo (por ejemplo, una unidad monetaria o número de víctimas por año, dado que la probabilidad de la amenaza presenta unidades de tiempo) (Escuder et al., 2010).

El mismo Escuder et al. (2010), sostiene que existe una gran variedad de herramientas para la estimación del riesgo de inundación o de alguno de sus componentes. Estas herramientas pueden dividirse en completas o parciales, según caractericen uno o dos componentes del riesgo. También pueden clasificarse como cuantitativas o cualitativas, según si obtienen o no un valor numérico para cuantificar la vulnerabilidad.

Para el presente trabajo en el sector del Puerto de El Encano del lago Guamués, no se cuenta con datos para estimar el riesgo de forma completa y cuantitativa, sin embargo, si se tiene información para poder definir el riesgo de manera completa y cualitativa. Se dispone de las series históricas de los niveles del lago para estimar cuantitativamente la probabilidad de ocurrencia de inundaciones, y se posee información para estimar de manera cualitativa las consecuencias, para lo que se tomará en cuenta la presencia o no de población y la exposición o no de 
infraestructura presente en el área de influencia del lago.

Además para la vulnerabilidad, se tendrá en cuenta el concepto de "Instalaciones críticas", que de acuerdo al Manual de Manejo de Peligros de la OEA/DDRMA (1993), es definido como "Todas aquellas instalaciones o mejoras hechas por el hombre, que por razón de su función, singularidad o tamaño, al ser destruidas, dañadas o al interrumpirse sus servicios por un evento catastrófico, pueden causar extensos daños a la propiedad y/o perturbar las actividades socio-económicas vitales (ejemplo: hospitales, estaciones de bomberos, etc.)". Es decir, para deducir cualitativamente la vulnerabilidad se adopta como criterios la presencia o no de población expuesta a las diferentes zonas de inundación, y la infraestructura combinada con la existencia o no de instalaciones críticas que puedan estar involucradas con dichas zonas, dando como resultado diferentes grados de vulnerabilidad, que no son una estimación cuantitativa de pérdidas de vidas, ni de pérdidas económicas, no obstante permite definir unos grados de vulnerabilidad de acuerdo a la exposición ante la amenaza de inundación de la población y la infraestructura, incluyendo las instalaciones críticas.

En la Figura 2, se indica el esquema que se desarrolla o aplica para la elaboración de los mapas de amenaza, vulnerabilidad y riesgo de inundación del área de estudio.

\section{Criterio hidrológico}

En la evaluación de la amenaza de inundación se parte de la ubicación o localización del evento natural y de su probabilidad de ocurrencia. Para
COMPONENTE FISICO

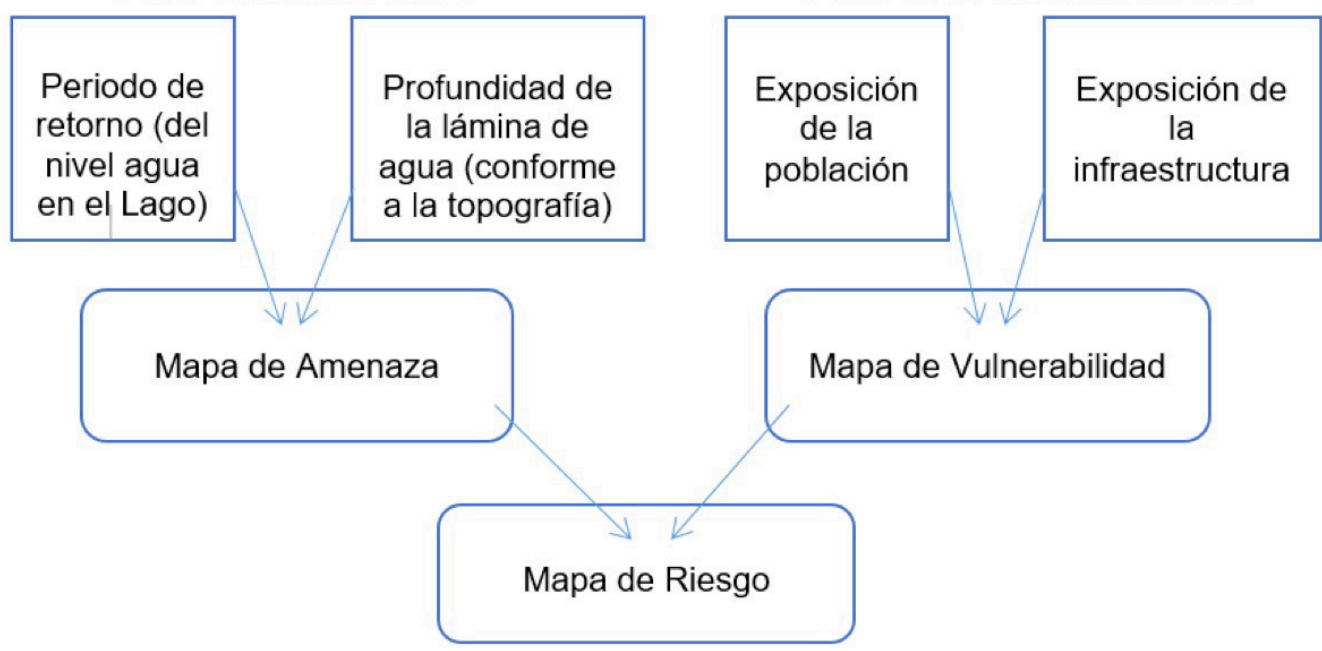

Figura 2. Esquema de elaboración de mapas de inundación. Fuente: Elaboración propia. 
determinar zonas inundables es necesario evaluar magnitudes de eventos probables o posibles (caudales, precipitaciones, niveles) en función de los tiempos de retorno que se adopten. Mediante la extrapolación de una ley de probabilidad deducida de la muestra de serie de datos hidrológicos, se definen las magnitudes máximas (eventos extremos) para áreas inundables en períodos de retorno determinados.

Los sistemas hidrológicos son afectados algunas veces por eventos extremos, tales como tormentas severas, crecientes y sequías. La magnitud de un evento extremo está inversamente relacionada con su frecuencia de ocurrencia, es decir, eventos muy severos ocurren con menor frecuencia que eventos más moderados. El objetivo del análisis de frecuencia de información hidrológica es relacionar la magnitud de los eventos extremos con su frecuencia de ocurrencia mediante el uso de una distribución de probabilidad (Chow et al., 1994).

Los estudios relativos a la determinación de eventos extremos han dado origen a leyes de distribución de probabilidad para representar la curva de frecuencia de una serie de eventos; entre las leyes que se utilizan habitualmente, están las leyes de Gumbel y Pearson III. Los métodos estadísticos utilizados para la estimación del nivel máximo están orientados a determinar la ley de distribución de probabilidad que mejor se ajusta a la serie de datos hidrológicos o registros existentes.

\section{Determinación de la ley de probabilidad teórica}

En primer lugar, se ajustarán a la curva de frecuencias experimental, las dos leyes probabilísticas propuestas, para la obtención de la ley de probabilidad teórica que mejor representa a la serie de niveles. El ajuste consiste en la determinación de los coeficientes propios de cada ley en función de los parámetros estadísticos de la serie (media, desviación estándar, coeficiente de asimetría).

Posteriormente, se analiza y gráfica, la calidad de las correlaciones obtenidas entre cada una de las leyes consideradas y la curva de frecuencias de la muestra, seleccionando aquella que provea la correlación más adecuada.

A continuación, se enuncian las fórmulas de las leyes de Gumbel y Pearson que se emplearon para los cálculos respectivos:

Distribución de valores extremos Tipo I Ley de Gumbel, estaley límite corresponde a una función de distribución de la forma:

$$
F(x)=P x \leq x_{1}=e^{-e^{-y}}
$$

Para Pearson III, se utiliza la función de frecuencia de la forma:

$$
x=\underline{x}+K . S
$$




\section{Resultados y Discusión}

La serie de niveles de la estación limnimetrica Sindamanoy en la laguna de La Cocha, cuenta con información diaria desde enero del año 1987 hasta diciembre del 2015 (29 años), sin embargo, los primeros seis años adolecen de información completa, principalmente en los meses de mayores precipitaciones y niveles altos del espejo de agua, por tanto, la serie de niveles máximos se tomaron a partir del año 1993 hasta el año 2015.

El promedio histórico de niveles de la laguna es de $267 \mathrm{~cm}$. El valor que aparece con mayor frecuencia de la serie diaria es el nivel de $274 \mathrm{~cm}$, el nivel mínimo presentado es de $185 \mathrm{~cm}$ y el nivel máximo $370 \mathrm{~cm}$, para un rango de $185 \mathrm{~cm}$ y una desviación estándar de 38,68.

La Figura 3, relaciona los datos de promedios mensuales de precipitaciones (Estación meteorológica Encano) y niveles (Estación limnimétrica Sindamanoy). De igual manera, se puede prestar atención a dos aspectos a analizar: en primer lugar, se observa que la curva de precipitaciones tiene un comportamiento básicamente bimodal, en cambio la curva de los niveles es monomodal; y en segundo lugar se aprecia que el pico de la curva correspondiente a los niveles se corre en el tiempo con respecto a la curva de precipitaciones, lo cual indica un retraso del pico de los niveles máximos con respecto al pico máximo de las lluvias.

Con relación al comportamiento bimodal de las precipitaciones y monomodal de niveles, es necesario anotar que en un lago o embalse como en un depósito, en la propagación de crecidas, el tránsito de niveles se puede atenuar y retardar; por otra parte los registros de precipitación y niveles en una estación del IDEAM diferente a la de El Encano, localizada a la salida del lago hacia el Sur, Estación Santa Isabel, tienen un comportamiento unimodal influenciado por la vertiente amazónica, lo cual contribuye para que los niveles del lago en la estación Sindamanoy se comporten de igual manera; no obstante a que el régimen de lluvias en la estación El Encano, localizada al norte del lago y más cercana al área de estudio tenga un comportamiento diferente , más bimodal, por encontrarse influenciada por la zona andina, los niveles presentan comportamiento unimodal.

Lo anterior sucede teniendo en cuenta que, Colombia está situada en el trópico del continente Americano, "como una isla entre tres cuencas de agua", si se piensa que además del Caribe y del Pacífico, Colombia recibe la influencia de la circulación atmosférica de la cuenca Amazónica, además la presencia de la barrera orográfica que constituyen los tres ramales de la cordillera de los Andes, induce la formación de climas locales y regionales de alta complejidad y dificultad de predicción a distintas escalas de tiempo (Poveda, 2004).

Las estaciones del IDEAM, afectadas por el sector andino, y por el sector amazónico, evidencian que el Lago Guamués concentra la influencia climática de estas dos zonas, tal como se expresa en el Plan de Manejo Humedal Ramsar (Corponariño, 2011), si 


\section{Promedios mensuales de precipitación - promedios mensuales de nivel}

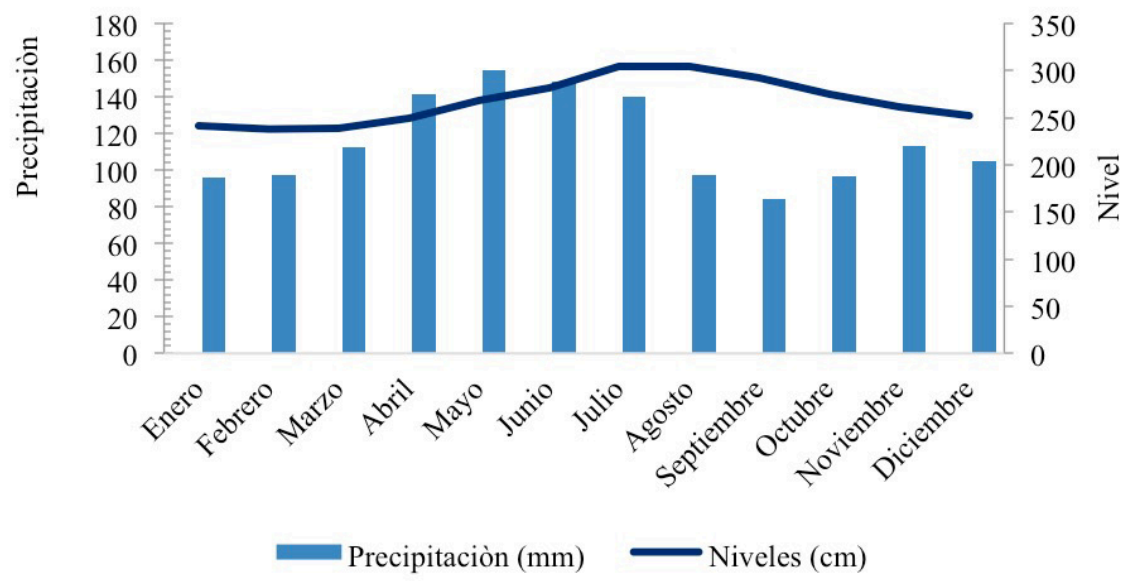

Figura 3. Relación precipitación Estación El Encano - niveles estación Sindamanoy (serie 1993 - 2015). Fuente: Elaboración propia.

bien en esta área la Zona de Confluencia Intertropical (ZCIT) produce lluvias, también se presenta un fenómeno de convección orográfica, que condiciona el establecimiento de un periodo lluvioso más intenso, debido al choque de los vientos alisios del sureste contra la vertiente oriental de la cordillera; debido a la disminución de la temperatura con la altura, las masas húmedas amazónicas se condensan, generando nubes de lluvia lo que hace que esta zona sea significativamente más húmeda que la existente en el frente de sotavento al lado occidental de la cordillera.

Corroborando lo anterior en la Figura 4, se observa la curva monomodal que recoge los datos de precipitación registrados en la estación Santa Isabel, acorde a la curva unimodal de niveles del río Guamués, comportamiento que también se observó en los datos de la estación limnimétrica Sindamanoy que refleja los niveles del lago; de igual manera se aprecia que los promedios mensuales de precipitación son mayores a los registrados en la estación El Encano (Fig. 3) a lo largo de todos los meses del año, donde para el mes de mayo la precipitación en El Encano es de $156 \mathrm{~mm}$, y para Santa Isabel es de $203 \mathrm{~mm}$, constatando que en la zona existen dos regímenes de precipitaciones y un régimen de niveles.

De manera complementaria, para la estación de El Encano los valores promedios mensuales multianuales de evaporación de mayo a septiembre oscilan entre 51,4 y 59,1 mm y son menores a los que se presentan en los meses de octubre a abril con valores mayores entre 66,8 y $88,3 \mathrm{~mm}$, factor que 
Promedios mensuales de precipitación y nivel Estación Santa Isabel 1993-2011

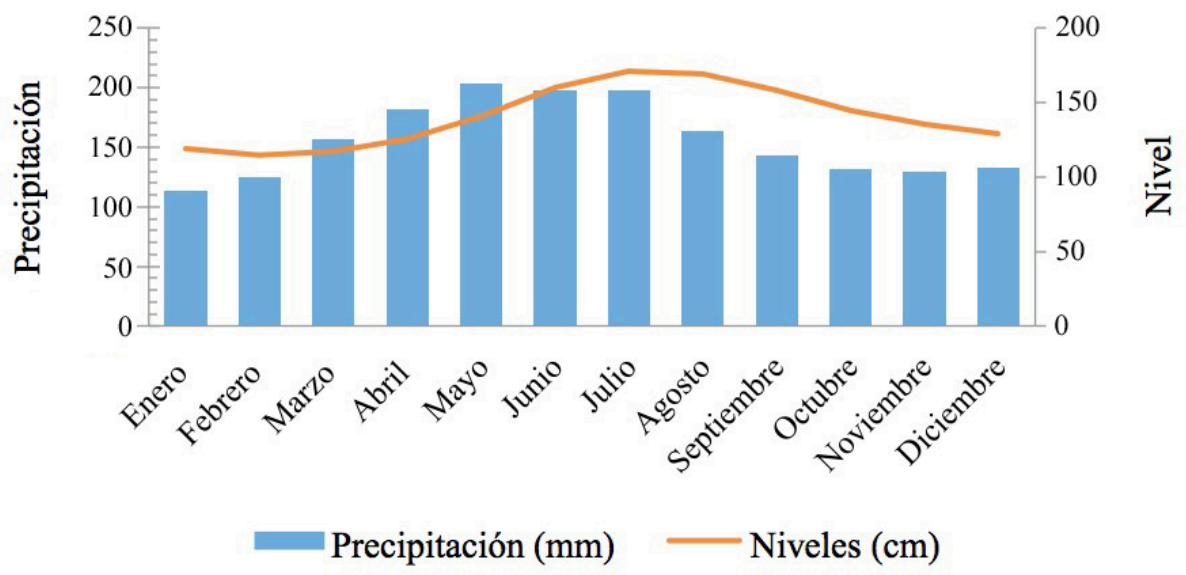

Figura 4. Relación precipitación - nivel (serie 1993 - 2011). Estación Santa Isabel. Fuente: Elaboración propia.

favorece que los niveles del lago en los meses de enero y febrero sean menores y el régimen anual de niveles tengan un comportamiento unimodal.

Con relación al segundo aspecto concerniente a la Figura 3, el desplazamiento en tiempo del pico de la curva de los niveles con respecto a la curva de precipitaciones, ocurre tanto para las estaciones de El Encano - Sindamanoy, como para la de Santa Isabel (Estación meteorológica y limnimétrica). Más allá que en términos generales un lago o embalse puede provocar la atenuación y/o el retraso de los picos de una crecida, este comportamiento y su razón para la cuenca del Guamués no se encuentra en la bibliografía, el cual puede deberse a diferentes factores: climáticos, geológicos, geomorfológicos, de suelos y cobertura vegetal.
En este sentido es necesario resaltar que geológicamente la cuenca es una zona compleja, en la que afloran diferentes tipos de rocas volcánicas y metamórficas y pueden presentar diferentes propiedades de permeabilidad e impermeabilidad que influyen de disímil manera en la infiltración y escurrimiento del agua, por ejemplo, dadas las características de foliación de las rocas metamórficas y los grados de meteorización de las rocas volcánicas. De igual forma la zona está atravesada por tres fallas con direcciones diferentes, las cuales se manifiestan con distinta intensidad en el fracturamiento y diaclasamiento de las rocas, que pueden intervenir en la dirección de flujos y en la permeabilidad de las mismas.

Por otra parte, se presenta una cobertura vegetal densa, en diferentes estratos y tipos de suelos que también pueden intervenir 
en la retención y filtración de agua de distinta manera.

Aunado a lo anterior, la cuenca del Guamués tiene una forma alargada que se demora más en concentrar la escorrentía que una cuenca con forma circular. El área que cubre la cuenca es de $477,1 \mathrm{~km}^{2}$ dividida en cuatro subcuencas, con una buena cantidad de microcuencas, entre las que se encuentra el río El Encano con un caudal de 2,4 $\mathrm{m}^{3} / \mathrm{s}$ (caudal aforado en octubre de 2016 por Corponariño), un área aproximada de 41,6 $\mathrm{km}^{2}$ de lago y un caudal de salida promedio histórico de $8,7 \mathrm{~m}^{3} / \mathrm{s}$.

Las microcuencas tienen diferentes comportamientos, sin embargo, no están instrumentadas, lo cual dificulta su estudio pormenorizado, en este sentido es necesario que en un futuro se aúnen esfuerzos institucionales para un mayor conocimiento de la cuenca y mayor detalle en el funcionamiento hidrológico de la misma.

\section{Determinación de la ley de probabilidad teórica}

Para el análisis de eventos extremos se empleó la serie de registros de niveles diarios confiables de 23 años (1993-2015) de la estación limnimetrica Sindamanoy.

En primer lugar, se ajustaron las leyes de Gumbel y Pearson III a la curva de frecuencias experimental, para luego contrastarlas y obtener la ley de probabilidad teórica que mejor representa a la serie de niveles.
Se calculó la media aritmética, la desviación standard y coeficiente de correlación. Los parámetros estadísticos de la serie encontrados son:

$\mathrm{X}^{1}=317,26$ (Media niveles)

$S=29,90$

$\mathrm{Cs}=0,04597$

$\mathrm{N}=23$ años

Una vez ajustadas las leyes de probabilidad de Gumbel y Pearson III, se analizó la calidad de las correlaciones de cada una de las leyes consideradas, comparando los valores teóricos y muestrales de las funciones de frecuencia relativa, para lo cual se utilizó la prueba de $\mathrm{X}_{\mathrm{c}}^{2}$ (chi - cuadrado).

Teniendo en cuenta que el valor de $X_{C}{ }^{2}$ para la distribución Pearson III es menor al de la distribución Gumbel y que gráficamente Pearson III visualiza una mejor tendencia al ajuste, se adopta la distribución Pearson III.

En la figura 5, se representan las curvas de frecuencia de la muestra, la ley de probabilística ajustada y los límites de confianza $=0,95$.

Adoptada la distribución Pearson III, se obtienen los niveles del lago Guamués en los diferentes períodos de retorno: uno, dos, cinco, 10, 25, 50, 75 y 100 años. 
Recurrencia - Niveles máximos anuales. Periodo de registro: 1993 - 2015 Estación Sindamanoy



— AJUSTE PEARSON $\quad$ NIVEL OBSERVADO —LIMITE SUPERIOR — LIMITE INFERIOR

Figura 5. Distribución de Pearson III. Fuente: Elaboración propia.

Relación de Nivel de escala -

Cota según recurrencia

El levantamiento topográfico del área de estudio se realizó con RTK(Real Time Kinematic) a escala detallada, en los planos las curvas de nivel se representan con una equidistancia de $10 \mathrm{~cm}$ y la superficie del relevamiento abarca una extensión de 50,3 hectáreas.

La proyección de los niveles sobre la planicie inundable, de acuerdo con la topografía del terreno, permite obtener zonas de inundación para un periodo de retorno fijado.
Parala determinación de las cotas de nivel, la parte superior de la regla limnimétrica de la estación IDEAM se la vinculó a las coordenadas y altitud de la placa PL1 ubicada en el hotel Sindamanoy, placa a la cual también se relacionó el levantamiento topográfico del área de estudio. En la Tabla 1, se indica la conversión de los niveles correspondientes a las recurrencias adoptadas a cotas sobre el nivel del mar (El nivel 400, cota 2.783,37 pertenece al tope superior de la regla limnimétrica).

El caserío Puerto de El Encano, se encuentra inmerso en la llanura de inundación de la laguna, que son superficies amplias de relieve 
plano-cóncavo, con fuertes encharcamientos por aguas delluvia, donde existe acumulación de aluviones finos y sedimentos orgánicos que permanecen saturados de agua durante gran parte del año.

Tabla 1. Cotas correspondientes a los niveles.

\begin{tabular}{ccc}
\hline $\begin{array}{c}\text { Recurrencia } \\
\text { (años) }\end{array}$ & $\begin{array}{c}\text { Nivel } \\
\text { (cm) }\end{array}$ & $\begin{array}{c}\text { Cota } \\
\text { (msnm.) }\end{array}$ \\
\hline 1 & 274 & $2.782,11$ \\
2 & 317 & $2.782,54$ \\
5 & 342 & $2.782,79$ \\
10 & 356 & $2.782,93$ \\
25 & 370 & $2.783,07$ \\
50 & 379 & $2.783,16$ \\
75 & 384 & $2.783,21$ \\
100 & 388 & $2.783,25$ \\
& 400 & $2.783,37$ \\
\hline
\end{tabular}

Análisis de amenaza

Para la definición de las zonas inundables, el valor adoptado como mínimo nivel de inundación es 274,00 cm, que corresponde a un tiempo de retorno de 1,1 años (se aproxima a un año), la cota calculada para este periodo es de $2.782,11 \mathrm{~m}$, que se puede considerar como la cota donde la laguna perdura la mayoría del tiempo, ya que se reitera con mayor frecuencia (las inundaciones ocurren cuando el nivel del agua alcanza cotas superiores). Para el mayor periodo de retorno considerado, esto es, 100 años, se calculó una cota de $2.783,25 \mathrm{~m}$.

La profundidad de la lámina de agua depende de la configuración topográfica del área inundada, correspondiente a rangos o intervalos de cotas paralos diferentes periodos de retorno adoptados. La profundidad de la lámina de agua será superior para las áreas con cotas más bajas, e inferior para las áreas con cotas más altas. La zona de amenaza mayor corresponde al área comprendida entre las cotas acordes a una frecuencia alta.

En la Tabla 2, se establecen los tres rangos de amenaza por inundación, cuyos valores asignados van de tres a uno, donde tres indica grado de amenaza alta y uno amenaza baja, y la equivalencia dos, valoración intermedia a las anteriores. A los grados finales se les

Tabla 2. Valoración de los rangos y grados de amenaza por inundación.

\begin{tabular}{ccccc}
\hline Recurrencia & Rangos & Valor & Grado & Color \\
\hline R (años) & Cotas (m s. n. m.) & Criterio & Amenaza & \\
\hline 1 a 5 & $2.782,11-2.782,79$ & 3 & Alto & Rojo \\
5 a 25 & $2.782,79-2.783,07$ & 2 & Medio & Naranja \\
25 a 100 & $2.783,07-2.783,25$ & 1 & Bajo & Amarillo \\
\hline
\end{tabular}


fija un color para poder representar en el mapa las diferentes zonas de peligro que se determinan para el área de estudio, rojo para amenaza alta, naranja para intermedia y amarilla para baja. El mapa resultante se indica en la Figura 6.

Zona de amenaza alta: Es la zona que se extiende desde la cota $2.782,11$ a $2.782,79$ msnm., abarcando un área de $0,24 \mathrm{~km}^{2}$, corresponde a un periodo de retorno entre uno y cinco años.

Zona de amenaza media: Esta zona se extiende desde la cota $2.782,79$ a $2.783,07$ msnm, involucra un área de $0,045 \mathrm{~km}^{2}$, acorde a un periodo de retorno entre cinco y 25 años.

Zona de amenaza baja: Es la zona que se extiende desde la curva de nivel 2.783,07 a $2.783,25 \mathrm{msnm}$, abarca un área de 0,05 $\mathrm{km}^{2}$, limitada a un periodo de retorno que comprende entre 25 y 100 años.

\section{Análisis de Vulnerabilidad}

Para analizar la vulnerabilidad se adopta como criterios para su evaluación la presencia o no de población en las zonas de inundación y la exposición de infraestructura combinada con la existencia o no de instalaciones críticas que puedan estar comprendidas en dichas zonas (Organización de Estados Americanos OEA, 1993), dando como resultado diferentes grados de vulnerabilidad.

Las calificaciones de los criterios población e infraestructura se suman para estimar el grado de vulnerabilidad del sector expuesto a la amenaza de inundación, como se indica en la matriz de la Tabla 3 y en la Figura 7 se muestra la infraestructura e instalaciones críticas expuestas, su área de influencia, y los diferentes grados de vulnerabilidad. Con los valores resultantes de la Tabla 3., se establecen los distintos grados de vulnerabilidad:

Tabla 3. Matriz de vulnerabilidad.

\begin{tabular}{|c|c|c|c|}
\hline Población & & $\begin{array}{c}\text { Sin } \\
\text { Población }\end{array}$ & $\begin{array}{c}\text { Con } \\
\text { Población }\end{array}$ \\
\hline & + & $\mathrm{O}$ & 1 \\
\hline \multicolumn{4}{|c|}{ Infraestructura } \\
\hline $\begin{array}{c}\text { Sin } \\
\text { instalación } \\
\text { crítica }\end{array}$ & 1 & 1 & 2 \\
\hline $\begin{array}{c}\text { Con } \\
\text { instalación } \\
\text { crítica }\end{array}$ & 2 & 2 & 3 \\
\hline
\end{tabular}

Vulnerabilidad alta: Para el área de estudio se define zona de grado 3, con presencia de población, infraestructura (Viviendas, vía, redes de energía eléctrica y acueducto) e instalaciones críticas representada por el sector de la escuela, el sector del muelle y casa de bomberos.

Vulnerabilidad media: Zona de grado 2, se presentan dos casos, uno con presencia de población e infraestructura sin instalaciones críticas, representada por la mayor parte del poblado o caserío; y otro caso sin población, con infraestructura y presencia de instalaciones críticas, representada por el 


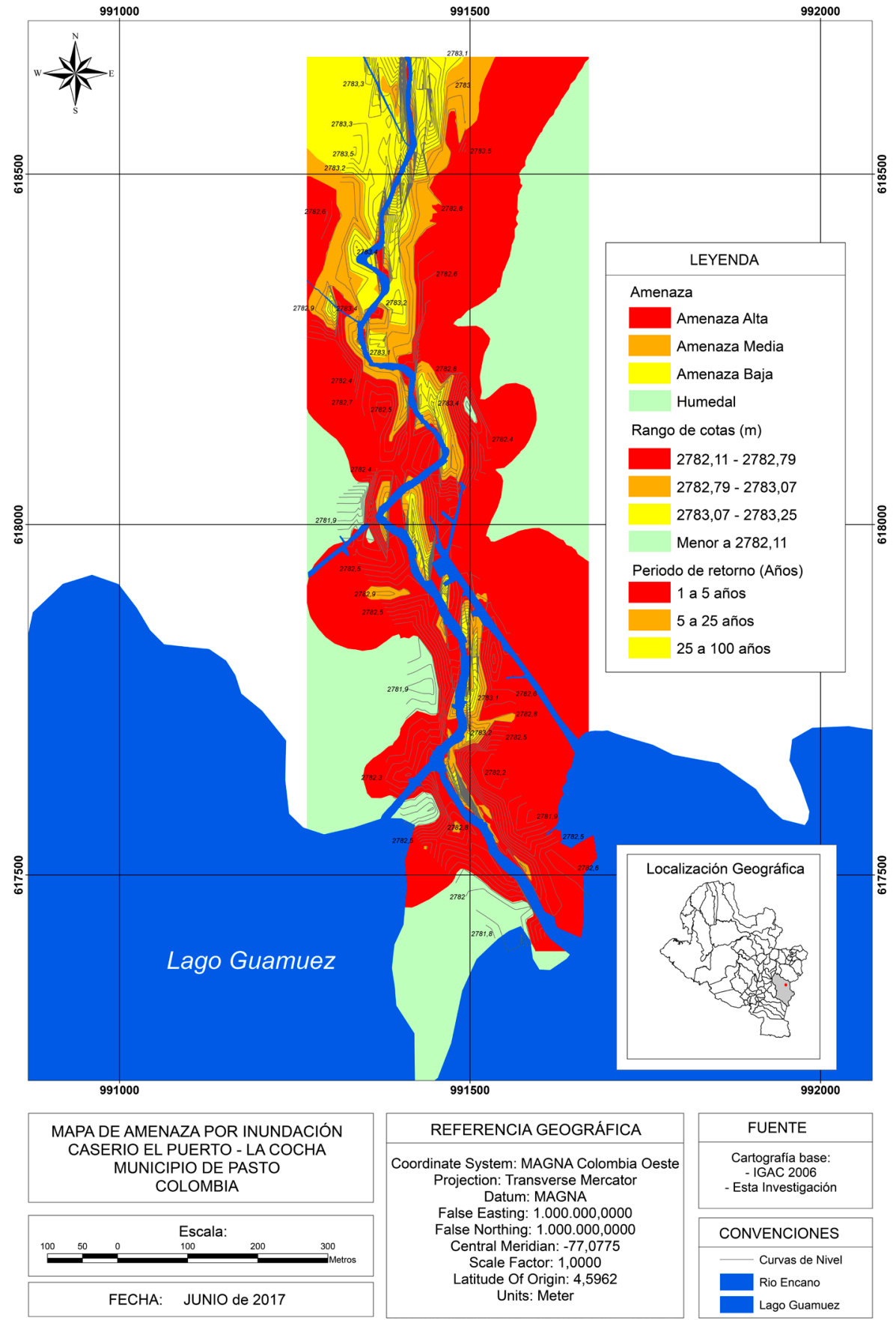

Figura 6. Mapa Zonificación de amenaza por inundación. 
sector de la capilla y salón comunal que son sitios de reunión y solo aglutina población en determinadas ocasiones.

Vulnerabilidad baja: Zona de grado 1, sin población y sin infraestructura, área que está sujeta a invadirse y poblarse, dada la tendencia al aumento demográfico y la demanda local de terrenos para infraestructura turística, la constituye el área de humedales que bordea las otras dos zonas.

\section{Análisis de riesgo}

De acuerdo a Escuder (2010), una vez determinados los mapas de amenaza por inundación y de vulnerabilidad, se integran para poder obtener el mapa de riesgo.

Aplicando la expresión $\mathrm{R}=\mathrm{A} * \mathrm{~V}$ se elabora una matriz donde se combinan los valores de las variables de amenaza y vulnerabilidad obtenidos en los Puntos anteriores y consignados en la Tabla 4.

Tabla 4. Determinación del riesgo.

\begin{tabular}{ccccc}
\hline $\begin{array}{c}\text { Valor de la } \\
\text { amenaza }\end{array}$ & Baja & Media & Alta \\
\hline $\begin{array}{c}\text { Grado de } \\
\text { Vulnerabilidad }\end{array}$ & $\mathbf{1}$ & $\mathbf{2}$ & $\mathbf{3}$ \\
Baja & $1^{\mathbf{0}}$ & 1 & 2 & 3 \\
Media & $2^{\mathbf{0}}$ & 2 & 4 & 6 \\
Alta & $3^{\mathbf{0}}$ & 3 & 6 & 9 \\
\hline
\end{tabular}

Zona de riesgo alto (A): Puede tener efectos potencialmente dañinos graves. Es la zona que pertenece al valor nueve, con una vulnerabilidad alta, con presencia de población, infraestructura e instalaciones críticas, que pueden afectarse de manera grave por estar expuestos a amenaza alta, abarca un área de $0,005 \mathrm{~km}^{2}$. En la figura 8 , se puede observar el mapa resultante de zonificación del riesgo.

Zona de riesgo medio (M): Puede tener efectos potencialmente dañinos moderados. Es la zona que corresponde al rango cuatro a seis, con una vulnerabilidad media con presencia de población e infraestructura que pueden afectarse de manera moderada a considerable, por estar expuestos a amenaza media a alta, abarca un área de $0,082 \mathrm{~km}^{2}$.

Zona de riesgo bajo (B): Puede tener efectos potencialmente dañinos leves. Es la zona que corresponde al rango uno a tres, con una vulnerabilidad baja sin presencia de población e infraestructura que puedan verse afectados por estar expuestos a amenaza baja a alta, abarca un área de $0,26 \mathrm{~km}^{2}$.

Esta metodología empleada para calcular el riesgo por inundaciones, no coarta o restringe los resultados para cuantificarlavulnerabilidad y el riesgo, sino que utiliza la información disponible y recurre a conceptos como el de instalaciones críticas o equipamientos básicos (como salud y educación que, prestan un servicio social y al afectarse amplifican los efectos del desastre), para poder evaluar y valorar cualitativamente la vulnerabilidad y combinarla con la estimación cuantitativa de 


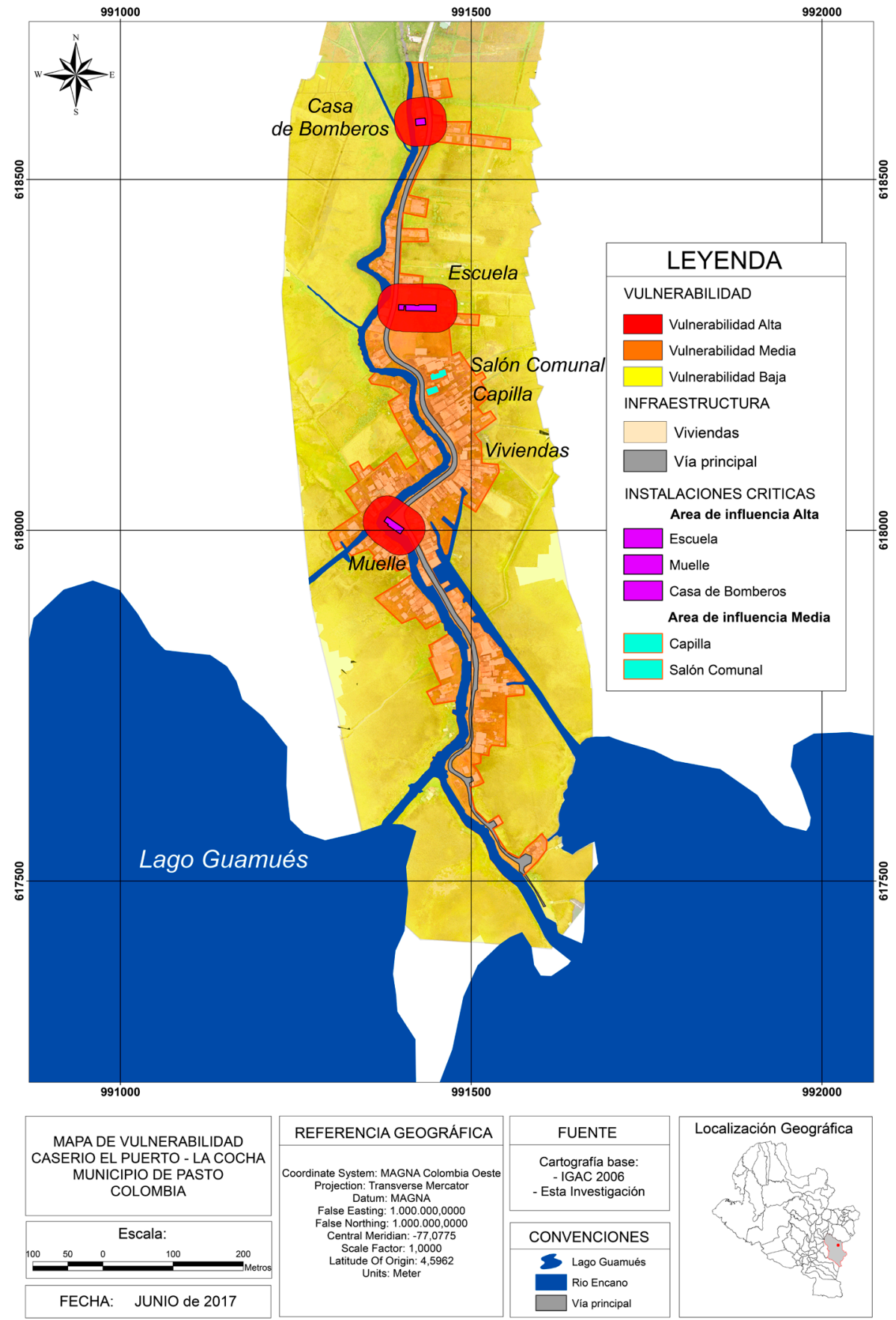

Figura 7. Mapa Zonificación de Vulnerabilidad. 


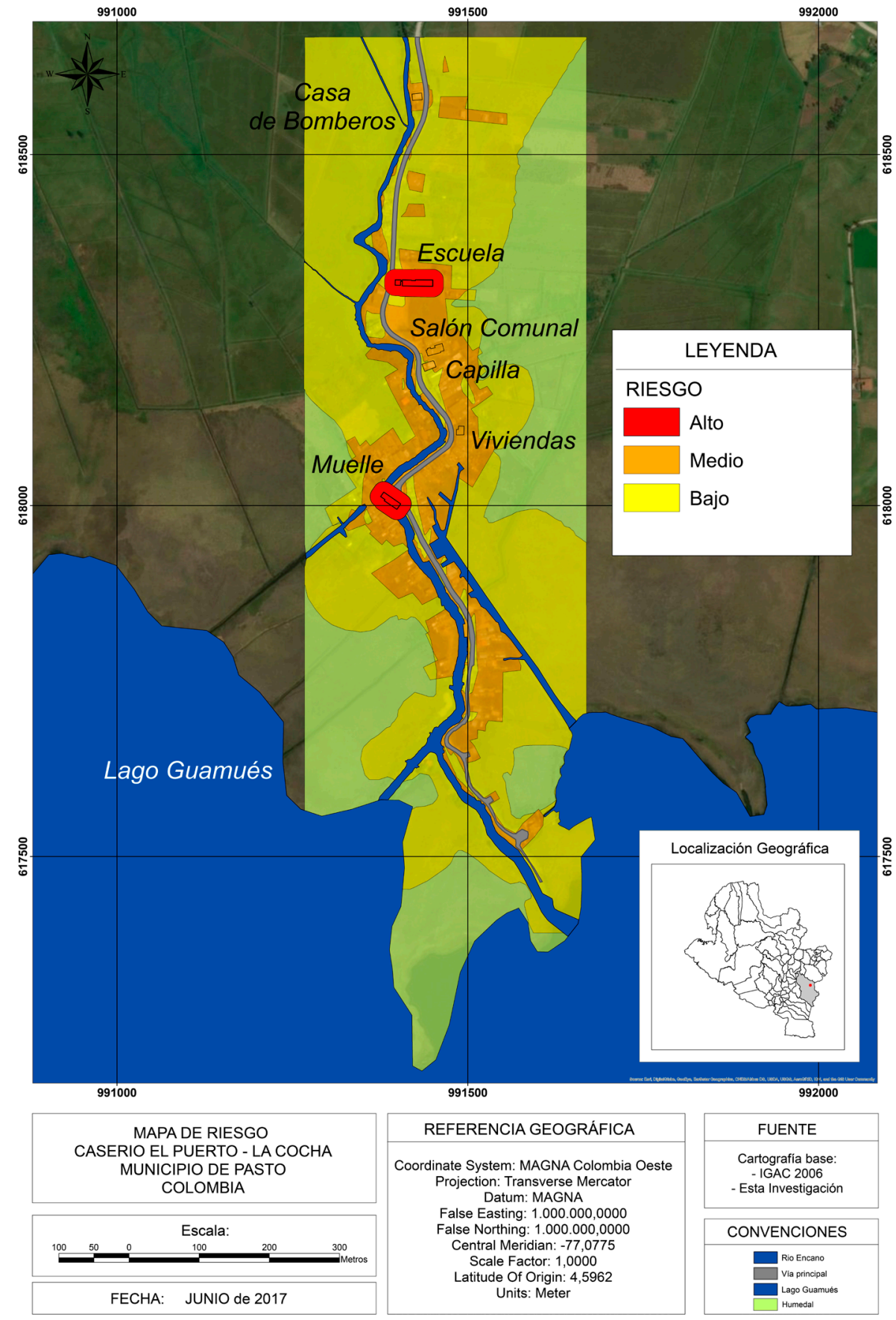

Figura 8. Mapa de zonificación de riesgo por inundación. 
la probabilidad de ocurrencia de inundaciones (amenaza); para así , obtener el riesgo de una manera completa y cualitativa. Metodología que de ninguna manera pretende estandarizar un único procedimiento, sino que se ajusta al detalle disponible.

\section{Conclusiones}

Una vez adoptada la distribución Pearson III por ajustarse mejor a la muestra, y considerando los períodos de retorno y las cotas calculadas, se delimitaron tres zonas de inundación en el caserío del Puerto: para un período de retorno con frecuencia alta, entre uno y cinco años, el rango de cotas es 2.782,11-2.782,79 msnm, zona catalogada como de amenaza alta; para un período de retorno con frecuencia media, entre cinco y 25 años, el intervalo de cotas es 2.782,792.783,07 msnm, clasificada como amenaza media y para un período de retorno con frecuencia baja, entre 25 y 100 años, el rango de cotas es 2.783,07-2.783,25 msnm, catalogada como zona de amenaza alta.

Con el objeto de generar medidas de prevención y ordenamiento territorial, a partir de la determinación de zonas de amenaza y vulnerabilidad, se estableció la zonificación y el mapa de riesgo por inundación en tres zonas: alta, media y baja. Se especificaron las diferentes zonas de riesgo: Alto (A), que puede tener efectos potencialmente dañinos graves sobre la población, infraestructura e instalaciones críticas, abarcando un área de $0,005 \mathrm{~km}^{2}$. Riesgo Medio (M), que puede tener efectos potencialmente dañinos moderados sobre la población e infraestructura, comprende un área de $0,082 \mathrm{~km}^{2}$. Riesgo Bajo (B), que puede tener efectos potencialmente dañinos leves, sin presencia de población e infraestructura, incluye un área de $0,25 \mathrm{~km}^{2}$.

Para la zona definida con alto riesgo, que abarca un área de $0,005 \mathrm{~km}^{2}$, se recomienda prohibir asentamientos de viviendas, y reubicar la escuela a un lugar fuera de las zonas de amenaza. El traslado o reasentamiento debe realizarse fuera de la llanura de inundación, hacia cotas más altas.

En las zonas determinadas como de medio riesgo: que son áreas que pueden afectarse de manera considerable a moderada, se aconseja prohibir más asentamientos de viviendas, y la reubicación del salón comunal y la capilla a un lugar más seguro.

Para la zona de bajo riesgo: área sobre la cual puede presentarse efectos potencialmente dañinos leves, se sugiere evitar que sea poblado para no aumentar el grado de vulnerabilidad y riesgo, y de la misma forma no extender el impacto ambiental negativo sobre el humedal y el lago.

\section{Agradecimientos}

A los directivos y docentes de la maestría en Ecohidrología de la Facultad de Ingeniería y la Facultad de Ciencias Naturales y Museo de la Universidad Nacional de La Plata de la República Argentina, por haber brindado la posibilidad de realizar este trabajo de tesis. 
A la Corporación Autónoma Regional de Nariño, a los funcionarios y personas que colaboraron en la construcción del presente estudio.

\section{Bibliografía}

Colombia. Congreso de Colombia. 1997. Ley 388 (Julio 18). Ley de Ordenamiento Territorial por la cual se modifica la Ley 9 de 1989, y la Ley 2 de 1991. Bogotá: Diario Oficial.

Colombia. Congreso de Colombia. 2012. Ley 1523 (abril 24). Por la cual se adopta la política nacional de gestión del riesgo de desastres y se establece el Sistema Nacional de Gestión del Riesgo de Desastres. Bogotá: Diario Oficial.

Corporación Autónoma Regional de Nariño - CORPONARIÑO. 2011. Plan de Manejo Ambiental Integral Humedal RAMSAR Laguna de La Cocha. Recuperado de www.Corponariño.com.co

Escuder, I., Matheu, E., \& Castillo, J. 2010. Análisis y evaluación de riesgos de inundación: estimación del impacto de medidas estructurales y no estructurales. Jornada CICCPV, Universidad Politécnica de Valencia, España. Recuperado de: http://www. edams.upv.es/docs/Ponencia_IE_EM_15O_Final_2010_10_05.pdf

Ghul. 1976. Geografía Colombiana. Bogotá: Instituto Geográfico Agustín Codazzi - IGAC.

Instituto Alexander Von Humboldt - IAvH, World Wild FundWWF Colombia y Asociación para el desarrollo campesino ADC. 2004. Proyecto de incentivos para la laguna de La Cocha como sitio Ramsar. Pasto. Nariño. Colombia. 1-89

Organización de Estados Americanos- OEA. 1993. Manual sobre manejo de peligros naturales en la planificación para el desarrollo regional integrado. Departamento de Desarrollo Regional y Medio Ambiente Secretaría Ejecutiva para Asuntos Económicos y Sociales, Organización de Estados Americanos. Recuperado de:http://www.oas.org/dsd/publications/Unit/oea65s/begin.htm 
Poveda, G. 2004. La hidroclimatología de Colombia: Una síntesis desde la escala interdecadal hasta la escala diurna. En: Rev. Acad. Colomb. Cienc.Vol XXVIII, Número 107.

Masgrau, L. R. 2004. Los mapas de riesgo de inundaciones: representación de la vulnerabilidad y aportación de las innovaciones tecnológicas. Documents d'Anàlisi Geogràfica. 43:153-171.

Ven Te Chow, Maidment, D., May, L., 1994. Hidrología aplicada. Bogotá: Nomos, S.A. 683 pp. 